\title{
INVESTIGACIONES
}

\section{Estudio de caso sobre la evaluación por competencias en Educación Artística: Aportaciones a la Educación Patrimonial}

\author{
Case Study on Competence-based Evaluation in Arts Education: \\ Contributions to Patrimonial Education
}

\author{
Inmaculada Sánchez-Macías, ${ }^{a}$ Iván Jorrín-Abellán ${ }^{b}$ \\ ${ }^{\text {a} F a c u l t a d ~ d e ~ E d u c a c i o ́ n ~ y ~ T r a b a j o ~ S o c i a l, ~ U n i v e r s i d a d ~ d e ~ V a l l a d o l i d ~}$ \\ Telf.: (34) 983184018. Correo electrónico: inmaculada.sanchez.macias@uva.es \\ ${ }^{b}$ Bagwell College of Education, Kennesaw State University \\ Telf.: (1) 4705783389. Correo electrónico: ijorrina@kennesaw.edu
}

\begin{abstract}
RESUMEN
En este artículo presentamos un estudio de caso centrado en analizar si la evaluación de los aprendizajes en el área de Educación Artística en Educación Primaria, refleja las directrices curriculares LOE-LOMCE para la evaluación por competencias en el área. El trabajo realizado arroja luz sobre multitud de aspectos relacionados con la evaluación de competencias en el área de Educación Artística. Entre ellos destacan los desajustes que existen en la concepción de la evaluación de los profesionales de la Educación Artística, su práctica evaluadora diaria y sus conexiones con el currículo oficial. Entendiendo la Educación Artística como piedra angular de la Educación Patrimonial (Fontal, 2016), en el trabajo que presentamos nos atrevemos a extrapolar las tensiones identificadas en la praxis metodológica de la Educación Artística en Educación Primaria, al ámbito de los debates teóricos que se suscitan tanto a nivel pedagógico como social/sociológico en el conjunto de la Educación Patrimonial.
\end{abstract}

Palabras clave: evaluación de aprendizajes, educación artística, educación patrimonial, estudio de caso.

\begin{abstract}
In this article we present a case study focused on analyzing whether the learning assessment in the area of Artistic Education in Primary Education, reflects the LOE-LOMCE curricular guidelines for the evaluation by competences in the area. The work carried out sheds light on several aspects related to the evaluation of competences in the area of Artistic Education. These include the mismatches that exist in the conception of the evaluation of the professionals of the artistic education, its daily evaluating practice and its connections with the official curriculum. Understanding artistic education as a cornerstone of heritage education (Fontal, 2016), in this work we dare to extrapolate the tensions identified in the methodological praxis of artistic education in primary education, within the scope of the theoretical debates that arise both at the pedagogical and social / sociological levels in the whole of Heritage Education.
\end{abstract}

Keywords: assessment, art education, heritage education, case study. 


\section{INTRODUCCIÓN}

El trabajo que presentamos trata de arrojar luz sobre la forma en que se están evaluando los aprendizajes en el área de Educación Artística en Educación Primaria. Para ello centraremos nuestra atención en analizar si los procesos de evaluación que se implementan en las aulas reflejan las directrices curriculares LOE-LOMCE ${ }^{1}$ para la evaluación por competencias en el área.

Según Efland (2002), la función del arte a través de la historia cultural de la humanidad ha sido la construcción de la realidad. La enseñanza artística ha ayudado a los alumnos a comprender su mundo social y cultural. Es por ello que apreciar el arte y las manifestaciones artísticas nos ha ayudado a entender mejor nuestro entorno, contextualizando los hechos que en él acontecen. De ahí la importancia de nuestro estudio: comprender cómo se evalúan los aprendizajes en la Educación Artística en la etapa de Primaria es imprescindible para el desarrollo integral y global de las personas, y como señala en el Boletín Oficial del Estado $\left(\mathrm{n}^{\circ}\right.$ 117, 2014), "la distribución de la Educación Artística facilitará al alumnado ir creciendo en su desarrollo, acompañando e interrelacionando conocimientos de otras áreas y fortaleciendo su desarrollo competencial, conformándose un aprendizaje globalizado" (p. 44559).

El objeto de estudio se centra, por tanto, en analizar si la evaluación de los aprendizajes que se está realizando en el área de Educación Artística refleja las directrices curriculares enfocadas a la evaluación por competencias en esa área. Para su análisis se ha optado por utilizar el método del Estudio de Caso, dado que nos interesa comprender en profundidad la realidad que nos ocupa.

Comenzaremos nuestro artículo con un marco teórico en el que se apoya nuestro estudio, y que plantea tres aspectos principales: un estado de la cuestión de la Educación Artística para situarnos en las teorías y aproximaciones que están influyendo en la actualidad en este ámbito de conocimiento; la relevancia, aproximaciones y formación inicial docente en y para la evaluación de los aprendizajes en estas asignaturas; y, por último, cómo se forma en España a los futuros profesionales que serán los responsables de impartir la docencia en Educación Artística. Tras el marco teórico, describimos el marco metodológico de nuestra investigación siguiendo los pasos de Jorrín-Abellán (2016) en su modelo para la generación de diseños de investigación de corte cualitativo, optando por el estudio de caso propuesto por Stake (1995), dado que se alinea bien con la cosmovisión constructivista aportada por los investigadores.

Finalizaremos con el análisis de los resultados y las conclusiones y reflexiones finales en las que aportamos luz sobre la evaluación de aprendizajes en Educación Plástica y su aportación a los debates teóricos de la Educación Patrimonial.

\section{MARCO CONCEPTUAL}

Tal y como se muestra en la Figura 1, el marco conceptual de sustento del estudio propuesto consta de tres componentes fundamentales: a) La Educación Plástica y el lugar secundario al que se la ha relegado en la Educación Primaria; b) la evaluación de aprendizajes basada

LOE: Ley Orgánica 2/2006, de 3 de mayo, de Educación.

Fuente: https://www.boe.es/buscar/pdf/2006/BOE-A-2006-7899-consolidado.pdf

LOMCE: Ley Orgánica 8/2013, de 9 de diciembre, para la mejora de la calidad educativa.

Fuente: https://www.boe.es/buscar/pdf/2013/BOE-A-2013-12886-consolidado.pdf 
en competencias (concepto de competencia y su sentido en el ámbito educativo actual); y, finalmente c) la formación de profesorado en nuestro sistema universitario y cómo influye este en la forma de evaluar del futuro maestro de E. Plástica. A continuación, pasamos a describir pormenorizadamente cada uno de estos componentes.

Figura 1. Marco conceptual del estudio

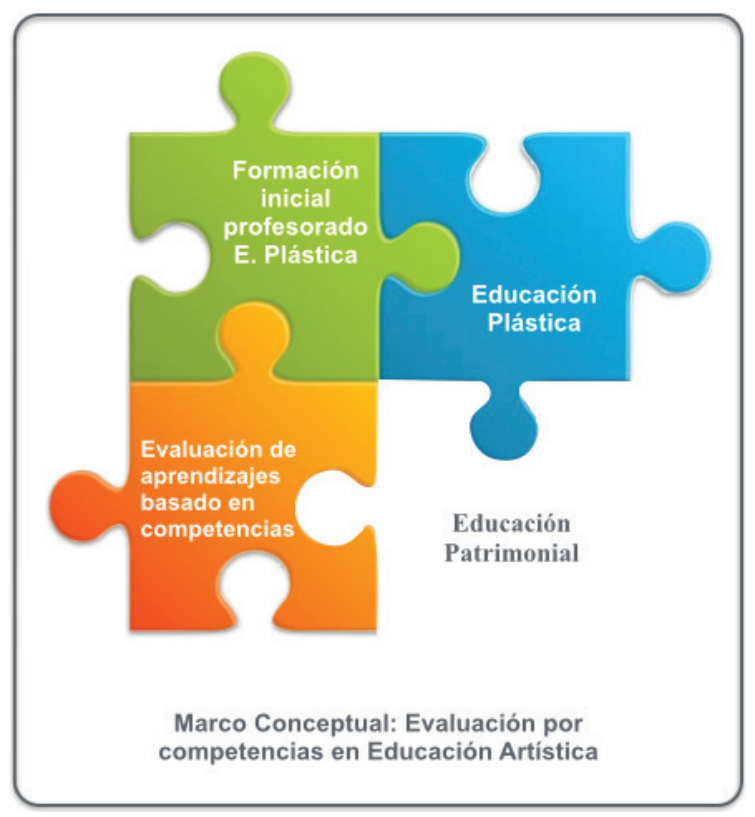

Fuente: elaboración propia.

Estos tres componentes mantienen una relación a modo de puzle en la que podemos apreciar que tanto la Educación Plástica como la Educación Patrimonial que la engloba, se ven influenciadas por la formación inicial del profesorado que se va a encargar en el futuro de la docencia de dichas asignaturas. El tercer componente muestra, a su vez, el impacto que la formación de los futuros maestros de Educación Plástica en Primaria, va a tener en su manera de evaluar los aprendizajes.

\subsection{EDUCACIÓN ARTÍSTICA PLÁSTICA}

Un breve recorrido histórico por las distintas concepciones de la asignatura que nos ocupa, nos guiará hasta la situación actual de la Educación Artística, para así poder comprender mejor la pertinencia, relevancia y necesidad del estudio que planteamos en este trabajo.

El final del siglo XX y principios del XXI se ven marcados por una evolución de la concepción de lo artístico en paralelo con los progresos y retrocesos sociales, las teorías y aproximaciones de otras disciplinas fuertemente relacionadas con el arte, como la psicología o la sociología. 
Desde finales del XIX, con Cizek en Viena, Marion Richardson en Inglaterra y sus homólogos en EE. UU. (Efland, 2002) y la definición de su expresión libre o autoexpresión creativa, la pedagogía del arte ha ido sufriendo numerosos cambios en consonancia con los modelos de sociedad y las legislaciones vigentes en cada momento. Bruner, en 1972, con su libro "Educando la Visión Artística" defiende la necesidad de un concepto amplio de Educación Artística, siendo precursor de lo que más tarde se conoce por Educación Artística como Disciplina (EACD), sugerido por Marín-Viadel (1987) (Discipline Based Art Education). La Educación Artística como disciplina fue concebida en 1984 por Dwaine Greer, antigua presidenta del Paul Getty Institute for Educators on the Visual Arts (DBAE). DBAE rompe con las concepciones anteriores porque trata de impartir una visión completa del arte mediante el estudio de cualquier obra determinada o tipo de trabajo por medio de cuatro disciplinas diferentes, adaptados a las edades y niveles específicos de calidad: producción del arte, historia del arte, crítica del arte y estética.

Unos años más tarde surge el currículo constructivista basado en los preceptos de Piaget (Piaget, Lorenz \& Erikson, 1992) y el currículo multicultural, con representantes como Kincheloe y Steinberg en su libro "Cambiando el multiculturalismo" (1997), hundiendo sus raíces en el postmodernismo, donde se pretende formar personas críticas con el sistema social, prestando especial atención a la diversidad cultural en el arte.

Durante los últimos años se ha ido desarrollando una diversidad de currículos como el reconstructivista, el posmoderno, la Teoría Crítica, la Pedagogía de Arte Crítica, la Educación Artística basada en la Cultura Visual (VCAE) con Freedman y su "Teaching visual culture: curriculum, aesthetics and the social life of art," y el más reciente currículo basado en la modernidad líquida, que se entiende como una época en la que la formación continua no debería dedicarse exclusivamente al fomento de las habilidades técnicas y a la educación centrada en el trabajo, sino, sobre todo, a formar ciudadanos que recuperen el espacio público de diálogo y sus derechos democráticos, pues un ciudadano ignorante de las circunstancias políticas y sociales en las que vive será totalmente incapaz de controlar el futuro de estas y el suyo propio (Bauman, 2007).

En la actualidad, la Educación Artística en España se encuentra en una situación en la que se está asistiendo a un cruce de propuestas que se mezclan entre sí, lo que da lugar a diversas teorías y prácticas artísticas encontradas. Estas propuestas de enfoques multiculturales, críticos y autocríticos proponen un currículo de la Educación Artística abierto a la pluralidad, flexibilidad, a la multiplicidad de manifestaciones artísticas, así como al desarrollo de colectivos minoritarios y de las artes populares.

Este hecho influye en nuestra investigación en un aspecto importante, ya que la confluencia de aproximaciones en la comprensión de la Educación Artística debería ser tenida en cuenta a la hora de orientar a futuros maestros hacia una visión más interdisciplinar del área. Así, la Educación Artística puede entenderse desde una visión integradora, fomentando una adecuada y necesaria conexión con otros conocimientos científico-técnicos, sin convertirse en una simple área instrumental. Para que esto ocurra, los implicados en el proceso educativo deben trabajar de forma conjunta, fomentando la integración de las diferentes disciplinas educativas, para brindar con ello la relevancia que los procesos de aprendizaje requieren (Salgado, 2012). Para ello, habría que tener muy presente la formación del profesorado que imparte las asignaturas relacionadas con la educación artística en nuestro país.

A este respecto, cabe señalar que la situación actual del área sigue alertando sobre cuestiones cronificadas, divididas entre las visiones históricas que minusvaloran el área al 
abogar por concepciones reduccionistas ("hacer dibujo" "hacer manuales"), y la falta de horas lectivas en el currículum de las áreas artísticas, aspecto este último que contradice los postulados teórico-epistemológicos defendidos en la actualidad, como mencionábamos anteriormente.

En la actualidad, en el área de Educación Artística en el currículo de Primaria en España sigue siendo el maestro generalista el que imparte la docencia (Bamford, 2006), o el graduado en Educación Primaria con mención generalista. El tiempo del que dispone para desarrollar el currículo (por ejemplo, en Castilla y León son dos horas semanales, al menos para E. Musical; o en Extremadura, son dos horas a la semana, una para E. Plástica y una para E. Musical), es muy escaso para llevar a cabo programas tan amplios y en los que los objetivos a trabajar son muy diversos (Robinson, 1999; Sharp \& Le Mátais, 2000), máxime cuando las horas lectivas están compartidas entre Plástica y Visual con la Educación Musical, asignaturas con lenguajes propios y distintos.

\subsection{EVALUACIÓN DE APRENDIZAJES EN EDUCACIÓN ARTÍSTICA}

Una vez presentada una aproximación histórica a la evolución del concepto de Educación Artística, resulta importante para nuestro trabajo centrarnos en la forma en que en la actualidad se evalúan los aprendizajes en el área. En esta sección nos acercaremos a la cuestión desde los planteamientos generales propios de la evaluación educativa, para posteriormente centrarnos en la evaluación de aprendizajes, aspecto crítico en nuestra investigación.

En el año 2000 se reúne el Consejo de Lisboa en sesión especial del Consejo Europeo, para reforzar la economía y la cohesión social europeas, con el objetivo estratégico de promover una conversión progresiva de la economía de mercado en una economía basada en el conocimiento, fomentando la modernización del estado de bienestar social y de los sistemas educativos. Es en este contexto donde se plantea el término de "competencia clave", como cualificación básica que se debe tener en cuenta en la educación y formación para la vida y el trabajo en la sociedad del conocimiento. El Consejo de Lisboa marca, por tanto, el inicio del marco común europeo sobre competencias. Posteriormente, como elaboración técnica dentro del Espacio Europeo de Educación Superior (EEES) se desarrolló el Proyecto Tunning (2003) ${ }^{2}$ promovido por un centenar de universidades de los países integrantes de la Unión Europea, con el objetivo de rediseñar los distintos currículos a partir de la citada Declaración de Bolonia. El Proyecto Tunning da importancia a las competencias, ya que responde con ellas a lo que debería saber, entender y ser capaz de hacer un estudiante para ser contratable. El cuestionario propuesto por el proyecto proponía tres tipos de competencias: instrumentales, interpersonales y sistémicas.

En nuestro trabajo se ha asumido la definición de "competencia" propuesta por Sánchez-Macías (2014) donde se definen como el conjunto de capacidades instrumentales y actitudinales, habilidades, aptitudes y disposiciones que se adquieren, se desarrollan y evolucionan para dirigir un proceso con el fin de solucionar ciertos problemas en cualquier aspecto de la vida.

Nos centramos a continuación en lo que se conoce en el mundo anglosajón como "assessment" o evaluación de los aprendizajes. A la evaluación, la Pedagogía tradicional

Disponibles en http://acreditacion.unillanos.edu.co/contenidos/cpacitacion_docente_2semestre_2007/ competencias_ proyectotuning.pdf 
la consideraba solo como un acto final desprendido de todas las acciones propias de la enseñanza y el aprendizaje, es decir, se tomaba únicamente en consideración la valoración de los productos finales del aprendizaje. Esta concepción estática y sumativa de la evaluación, pone en evidencia un concepto de educación también estática y centrada en los productos, no en los procesos. Una de las razones que justifica este hecho viene ocasionada por la confusión que en el mundo de lo educativo observamos entre los conceptos de evaluación y calificación. Esta confusión se ha reforzado como consecuencia de la influencia ejercida por las tendencias sociales neoliberales y su repercusión directa en los sistemas educativos, donde la acreditación de resultados se convierte en necesidad para el acceso al mundo laboral.

Asimismo, resulta importante recordar que la evaluación de aprendizajes, en su versión más tradicional, se apoya fuertemente en una comprensión clasista de los sistemas educativos, donde la evaluación se convierte en instrumento de selección social.

En la actualidad, observamos que la concepción de la evaluación de aprendizajes se ha visto afectada por numerosas aproximaciones teóricas provenientes de campos diversos, este es el caso de: a) las teorías sobre la empleabilidad del educando (Formichella \& London, 2005); b) los avances de la pedagogía contemporánea hacia modelos participativos; c) la incorporación de las TIC (Mishra \& Koehler, 2006); d) las aportaciones de la evaluación ecológica (Tikunoff \& Griffin, 1979) y finalmente; e) las aportaciones de la evaluación interdisciplinar (Legrand, 1970).

Estas aproximaciones teóricas aportan una base necesaria para comprender la evolución del concepto de evaluación a lo largo de los últimos años. No obstante, la evaluación de aprendizajes tiene sus raíces teóricas en la psicología cognitiva, y especialmente en la definición que Vigotsky ofreció de la zona de desarrollo próximo (ZDP). Esta se entiende como

la distancia en el nivel real de desarrollo, determinado por la capacidad de resolver independientemente un problema, y el nivel de desarrollo potencial, determinado a través de la resolución de un problema bajo la guía de un adulto o en colaboración con otro compañero más capaz. (Vigotsky, 1988, p. 133).

Es decir, la aplicación de esta aproximación nos lleva a plantear una evaluación centrada en los procesos, que se convierte, por tanto, en herramienta clave para el desarrollo del aprendizaje.

En segundo lugar, la concepción moderna de la evaluación también se apoya en las teorías sociológicas de la educación, centradas en la dimensión social del hecho educativo entendido en los varios círculos de influencia desde y hacia la sociedad circundante al centro educativo. Podemos mencionar a este respecto la escuela teórica anglosajona de Reino Unido y EE. UU., que resalta el enfoque en el desarrollo específico de cada aprendizaje "situado" (Lave \& Wenger, 1991) en un contexto, primando su evolución particular sobre la importación de patrones o herramientas externas de evaluación "prefabricadas". En consonancia resaltan ese concepto educativo dinámico por encima de las técnicas específicas del propio assessment. Como escuela teórica de esta concepción moderna de la evaluación, destaca la Assessment for Learning (AFL).

Por su parte, la escuela francófona (Canadá, Suiza) ha incidido sobre el concepto de contrato didáctico, en que cada parte educativa, profesor y alumnos pactan, según 
sus expectativas y objetivos, el desarrollo de los aprendizajes. Se percibe la evaluación como una regulación constante influenciada por los intereses particulares que aporta cada alumno a la misma. Como autor de esta escuela destaca Perrenoud (2009), quien defiende unos principios generales educativos como son la educación continua, la importancia del feedback y la intercomunicación. Entiende por tanto la evaluación como un hecho contextual, valorando los procesos por encima de los logros y planteando diversos instrumentos de evaluación y de diálogo.

Esta escuela, con sustento teórico en el Learning by doing de Dewey (1900), propone la utilización de técnicas específicas de apoyo a la evaluación de los aprendizajes, como el caso de estudio, y la solución de problemas concretos. También plantea el uso de ciertos elementos de monitorización de los avances y logro de objetivos. Para ello plantea el uso de numerosas técnicas de recogida de información para la evaluación del aprendizaje que se utilizan en la actualidad, como por ejemplo el uso de la observación sistemática, las rúbricas, los portafolios, las entrevistas, cuestionarios, cuadernos de clase, informes; así como el empleo de tecnologías mediante el uso del e-portafolio, las e-rúbricas, Learning Analytics, y el peer and on-line assessment.

Estas propuestas se alinean bien con los requisitos y demandas de la evaluación por competencias, aunque demandan un gran esfuerzo y formación específica de los docentes.

\subsection{FORMACIÓN DE PROFESORADO E INFLUENCIA EN LA FORMACIÓN DE FUTUROS MAESTROS}

La formación inicial de los futuros docentes de Primaria viene marcada por la Ley Orgánica de Educación (LOE), en la que se establece que para ejercer la docencia de las enseñanzas artísticas será necesario estar en posesión del título de Licenciado, Ingeniero o Arquitecto, o del título de Grado correspondiente en la Educación Primaria. También se reconoce la habilitación de otras titulaciones que pudiera establecer el Gobierno para determinados módulos. También menciona que excepcionalmente se podrá incorporar a profesionales, atendiendo a su cualificación y a las necesidades del sistema educativo, no necesariamente titulados, de nacionalidad extranjera (Ley Orgánica 2/2006 de Educación. Título III, Capítulo II "Profesorado de las distintas enseñanzas" y Capítulo III "Formación del profesorado"). Como vemos, las orientaciones proporcionadas por la legislación vigente a este respecto establecen que casi cualquier profesional con formación artística puede convertirse en docente del área, salvo en el caso de la Educación Primaria, donde se requiere la titulación de maestro.

En el terreno que nos ocupa, cabe mencionar que el futuro maestro suele enfrentarse al estudio de la materia con unas concepciones básicas y a veces erróneas, que determinarán su aprendizaje en los años de formación para la docencia, así como el aprendizaje de sus alumnos cuando ejerza su práctica profesional. Esta falta de especialización del profesorado, unida a los amplios objetivos definidos en el área, provoca en muchas ocasiones que los docentes no sepan qué enseñar y cómo hacerlo (Eisner, 1995).

Desde nuestro punto de vista, la situación en las Escuelas y Facultades de Magisterio de nuestro país es precaria en lo referido a la formación de futuros maestros en el área de Educación Artística. Para poder enseñar a los futuros docentes cómo fomentar los aprendizajes en el área de educación artística, deberían haberse formado previamente de manera holística, habiéndose ejercitado en las distintas dimensiones, destrezas, y habilidades que implica el área, aspecto que no se tiene en cuenta en las universidades españolas. 
Por un proceso natural, las capacidades y competencias que se desarrollan con la Educación Artística, concretamente con la Educación Plástica en Primaria, se frenarán si no se educan o se siguen desarrollando de una forma adecuada. Si los maestros que forman en esta etapa no están preparados para ello, provocarán un estancamiento del desarrollo de las habilidades artístico-creativas de los niños, como ha ocurrido con la mayoría de los adultos que no han recibido apoyo en el campo de la Educación Plástica y Visual cuando fueron niños. Por tanto, resulta clara la influencia que la formación inicial del profesorado en el área tiene en el futuro desarrollo de las generaciones venideras.

Los tres elementos desarrollados en esta segunda sección configuran el marco conceptual de sustento del estudio que presentamos a continuación. La evolución de la concepción de la Educación Plástica y el lugar secundario al que se la ha relegado en la Educación Primaria, la evolución de la evaluación de aprendizajes basado en competencias y, finalmente, las carencias en la formación inicial de futuros maestros, constituyen la base de los argumentos de sustento de la necesidad y relevancia de nuestro estudio.

\section{METODOLOGÍA}

En este apartado describimos pormenorizadamente la metodología empleada en el estudio desarrollado. Para ello seguiremos los pasos definidos por Jorrín-Abellán (2016) en su modelo para la generación de diseños de investigación de corte cualitativo.

\subsection{APROXIMACIÓN GENERAL DE INVESTIGACIÓN}

Resulta importante definir, en primera instancia, la aproximación general seguida en la investigación que hemos llevado a cabo. Dentro de las tres principales aproximaciones existentes en el campo de las ciencias sociales (cuantitativa, cualitativa y métodos mixtos), hemos optado por una aproximación interpretativa de corte cualitativo. Por ello, la finalidad última del trabajo realizado no pasa por explicar el fenómeno objeto de estudio, sino que, por el contrario, pretendemos alcanzar una comprensión profunda del mismo.

Por tanto, el estudio realizado pretende comprender si la evaluación que en la actualidad se desarrolla de los aprendizajes en el área de Educación Artística en Educación Primaria refleja las directrices curriculares LOE-LOMCE para la evaluación por competencias en el área.

\subsection{POSICIONAMIENTO DEL INVESTIGADOR O COSMOVISIÓN}

Tal y como menciona Creswell (2013), los investigadores cualitativos aportan a sus estudios, de forma ineludible, su particular manera de entender cómo funcionan las cosas en nuestro mundo y la forma en que se construye el conocimiento (Worldview). La dimensión epistemológica y ontológica que aporta el investigador influirá fuertemente en las decisiones metodológicas que tome en la fase de diseño de sus investigaciones. Por ello, resulta de vital importancia definir y reconocer nuestra forma de entender el mundo.

La aproximación interpretativa que hemos seguido se alinea con la cosmovisión de corte constructivista que como investigadores aportamos al proceso. Siguiendo esta cosmovisión, defendemos la construcción social del conocimiento fruto de la interacción social. 
De igual forma entendemos que el propio contexto social en el que se ha desarrollado nuestro estudio ha condicionado las interpretaciones que hemos realizado, y que por ello nos posicionamos subjetivamente ante el fenómeno objeto de estudio. Por este motivo, el peso de la investigación desarrollada, de corte constructivista, recae en los significados que sobre el fenómeno social estudiado han construido los participantes en la misma. De esta manera, los investigadores tan solo hemos interpretado los significados que nuestros participantes han generado.

\subsection{TRADICIÓN DE INVESTIGACIÓN}

De entre las principales tradiciones de investigación que encontramos en investigación cualitativa, hemos optado por emplear el estudio de caso, dado que nos permite comprender en profundidad un fenómeno en funcionamiento, delimitado espacio-temporalmente.

En el estudio de casos existen a su vez distintas conceptualizaciones, tal y como identifica Yazan (2015). De ellas optamos por la propuesta de Stake (1995), dado que se alinea bien con la cosmovisión constructivista aportada por los investigadores.

Tal y como describiremos en las secciones siguientes, nuestro estudio vendrá delimitado por la experiencia que como estudiante ha tenido la investigadora principal. Por ello el estudio se ha desarrollado en la Facultad de Educación y Trabajo Social de la Universidad de Valladolid (centro en el que la investigadora principal ha cursado estudios de grado y máster); un $\mathrm{CRA}^{3}$ en la localidad de Zaratán (centro en el que la investigadora realizó el Prácticum I); un instituto de Educación Secundaria en Plasencia (centro donde trabaja un compañero de Bellas Artes como profesor); y un centro de Educación Primaria en la localidad de Llerena (Badajoz) (centro donde cursó la EGB la investigadora).

\subsection{ESTRUCTURA CONCEPTUAL DEL CASO}

A continuación, desgranamos los componentes del estudio de caso desarrollado, conforme al esquema de estructura conceptual definido por Stake (1995) (Ver Figura 2).

El círculo central rodeado de una línea gruesa plantea los límites del caso mencionados en la sección anterior, así como las principales características del estudio (por ejemplo, funciones, lugares de actividad, informantes, técnicas de recogida de datos, etc.).

Del círculo central parten dos semicírculos que nos permiten definir los contextos del caso que influyen en sus raíces y comprensión; y, por último, en la base del círculo encontramos las tensiones de investigación (issues), los tópicos y las preguntas informativas que nos han permitido iluminar la complejidad del fenómeno estudiado.

De entre el conjunto de elementos estructurales que conforman el estudio de caso, nos centraremos a continuación en la descripción de sus funciones, los informantes, las técnicas de recogida de datos empleadas, las tensiones de investigación y las estrategias para el análisis de los datos.

La función principal del estudio fue la de intentar comprender si la evaluación por competencias, que en la actualidad se viene desarrollando en el área de Educación Artística en Educación Primaria, se alinea con las directrices curriculares establecidas en la legislación vigente.

C.R.A: centro rural agrupado. 
Para ello definimos una tensión de investigación central (o issue) de la siguiente forma: ¿Refleja la evaluación de los aprendizajes que se realiza en Educación Artística en el aula de Primaria las directrices curriculares enfocadas a la evaluación competencial en esa área? Esta tensión se centra eminentemente en el análisis de lo que sucede en la realidad de las aulas en relación con la evaluación por competencias. Al definirla teníamos en mente el documento puente que el profesorado elabora para vincular la normativa vigente con sus prácticas de aula. Los indicadores de logro que en él se definen van a permitir a las docentes definir unos indicadores de logro para posteriormente poder evaluar las competencias mostradas por su alumnado al desarrollar el currículo. Es decir, la intención era averiguar si lo que los currículos proponen es lo que se realiza en el aula en cuanto a la evaluación de aprendizajes.

De la tensión definida surge el siguiente aserto de investigación que se debe verificar posteriormente mediante el análisis de los datos: consideramos que en el aula de Educación Artística de Primaria no se realiza evaluación de aprendizajes por competencias según se propone en el currículo oficial.

Para poder iluminar la tensión planteada y así dar respuesta al aserto anterior definimos una serie de temas de interés, declaraciones temáticas o tópicos que nos permitieran enfocar la recogida y el análisis de los datos. Estas habilitarán la definición de un primer esquema de categorías de análisis de los datos. En el estudio establecimos las siguientes:

a. Formación inicial y profesional de los informantes: esta declaración temática pretende indagar en las relaciones existentes entre la formación de los maestros y profesores entrevistados y su elección para la enseñanza de artes plásticas, así como las influencias de su formación en su lenguaje plástico.

b. Las competencias docentes y artísticas de los informantes: basándonos en la definición de competencia propuesta por Sánchez-Macías (2014), esta declaración temática nos ayuda a valorar el conjunto de competencias que el profesorado debe desarrollar a nivel artístico y docente para la enseñanza de esta disciplina.

c. Estrategias de evaluación del aprendizaje artístico: mediante esta declaración pretendemos analizar las estrategias que puede poner en marcha el profesorado para realizar evaluación de aprendizajes en la educación artística. Más concretamente, valoramos si los docentes emplean estrategias propias o si, por el contrario, utilizan estrategias propuestas por otros.

d. Currículo en Educación Artística: esta declaración temática se definió con el objeto de analizar los currículos publicados hasta la fecha, concretamente sobre la plástica en Educación Artística en la etapa de Primaria. Es importante este tópico porque alrededor de él surgen ramificaciones que aclaran competencias, evaluaciones y otros aspectos de la docencia.

Los informantes y las técnicas de recogida de datos empleadas en el estudio se resumen en la Tabla 1.

En lo relativo a las estrategias empleadas para el análisis de los datos, cabe mencionar que seguimos un proceso de codificación abierta de datos mediante el uso de la herramienta de análisis Nudist*Vivo 10. En primer lugar, comenzamos analizando la autoetnografía narrativa generada por la autora principal del estudio, desde las categorías de análisis etic marcadas por las cuatro declaraciones temáticas propuestas en el estudio. Este análisis 
arrojó un conjunto de categorías de análisis de carácter emic, que posteriormente se aplicaron al análisis del resto de entrevistas. La Tabla 2 muestra las categorías de análisis emanadas del proceso.

Figura 2. Estructura Conceptual del Estudio de Caso

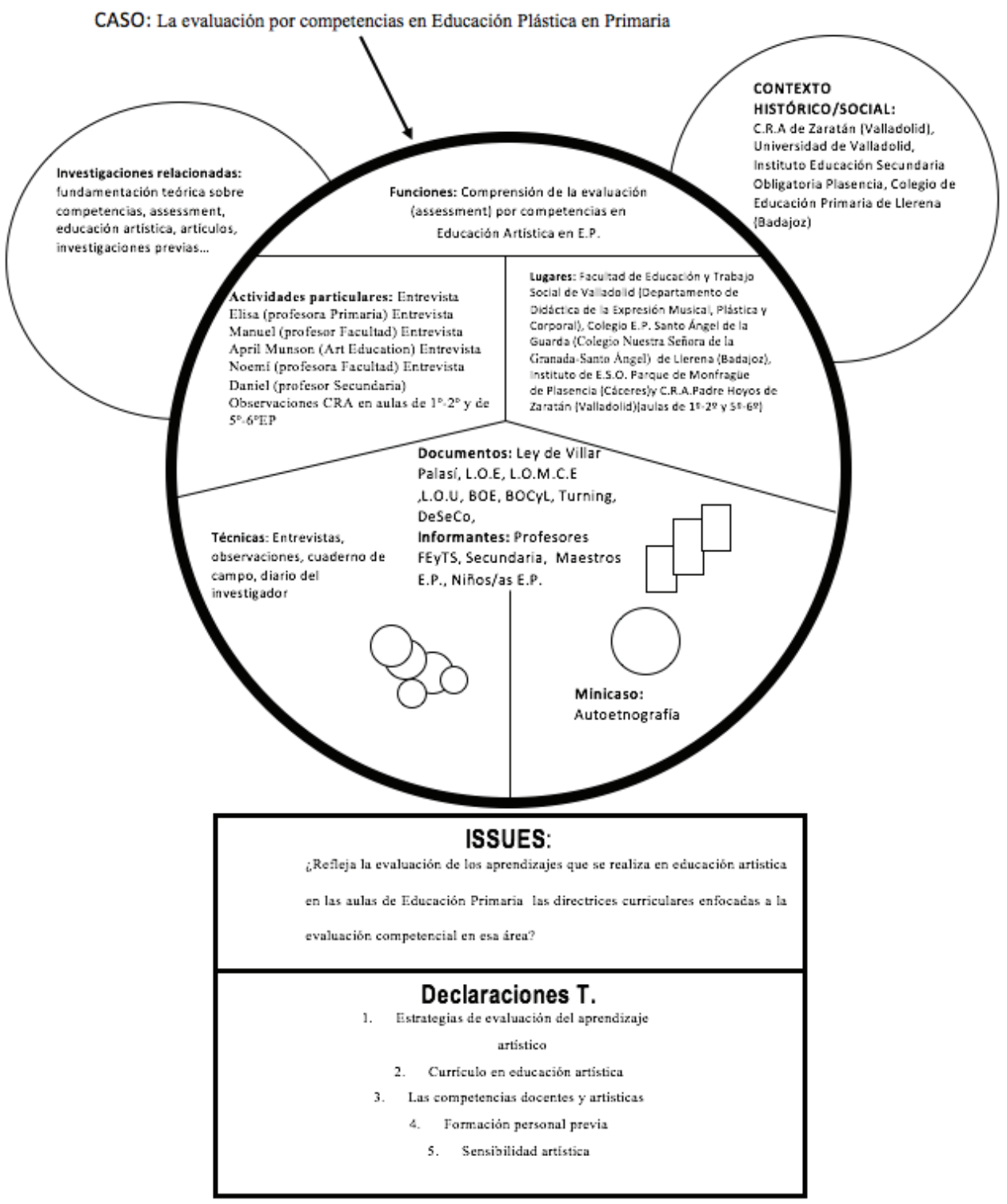

Fuente: elaboración propia. 
Estudios Pedagógicos XLIII, N 4: 229-250, 2017

ESTUDIO DE CASO SOBRE LA EVALUACIÓN POR COMPETENCIAS EN EDUCACIÓN ARTÍSTICA: APORTACIONES A LA EDUCACIÓN PATRIMONIAL

Tabla 1. Fuentes y técnicas de recogida de datos

\begin{tabular}{|c|c|c|c|}
\hline Técnicas & Fuentes e Informantes & Cantidad & Mes/Periodo/Año \\
\hline Entrevistas & 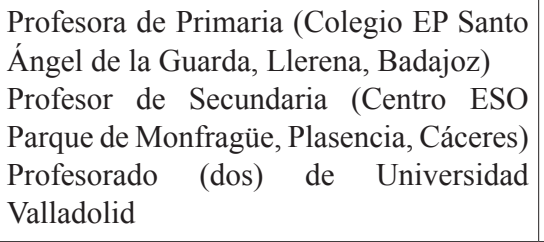 & $\begin{array}{l}1 \\
1\end{array}$ & $\begin{array}{l}\text { Diciembre } 2013 \\
\text { Mayo } 2014 \\
\text { Marzo, Abril y } \\
\text { Mayo de } 2014\end{array}$ \\
\hline Observaciones & $\begin{array}{l}\text { Aula de } 1^{\circ}-2^{\circ} \text { EP } \\
\text { Aula de } 5^{\circ}-6^{\circ} \text { EP } \\
\text { CRA Padre Hoyos de Zaratán, Valladolid }\end{array}$ & $\begin{array}{l}2 \\
2\end{array}$ & Mayo 2014 \\
\hline Consulta Documentos & $\begin{array}{l}\text { Biblioteca, profesorado universitario } \\
\text { (Uva) }\end{array}$ & 4 & $\begin{array}{l}\text { Noviembre 2013- } \\
\text { Mayo } 2014\end{array}$ \\
\hline $\begin{array}{l}\text { Cuaderno de campo } \\
\text { (autoetnografía) }\end{array}$ & Investigadora & 1 & $\begin{array}{l}\text { Diciembre 2013- } \\
\text { Mayo } 2014\end{array}$ \\
\hline
\end{tabular}

Fuente: elaboración propia.

Tabla 2. Categorías de análisis Étic y Émic

\begin{tabular}{|l|l|}
\hline \multicolumn{1}{|c|}{ Categorías Étic } & \multicolumn{1}{c|}{ Categorías Émic } \\
\hline 1. Formación personal previa & $\begin{array}{c}\text { 1. Formación } \\
\text { formaciónlprofesorado } \\
\text { formaciónlalumnado } \\
\text { formaciónlartista } \\
\text { formaciónlcreatividad } \\
\text { formaciónlsensibilidad } \\
\text { formaciónlestrategias comunicativas }\end{array}$ \\
\hline 2. Las competencias docentes y artísticas & 2. Familia y entorno \\
\hline 3. Estrategias de evaluación del aprendizaje artístico & 3. Estrategias de evaluación \\
\hline 4 .Currículo en educación artística & $\begin{array}{l}\text { 4. Currículolcorporal } \\
\text {-currículolmúsica } \\
\text {-currículolplástica }\end{array}$ \\
\hline
\end{tabular}

Fuente: elaboración propia. 


\section{PRINCIPALES RESULTADOS DEL ESTUDIO DE CASO}

Por motivos obvios de espacio, en esta sección nos centraremos en mostrar algunos de los principales resultados obtenidos dentro de cada una de las cuatro declaraciones temáticas que han guiado el análisis.

\subsection{FORMACIÓN INICIAL Y PROFESIONAL DE LOS INFORMANTES}

Dentro de esta primera declaración temática hemos obtenido tres resultados fundamentales.

En primer lugar, el profesorado universitario y de secundaria entrevistado hace referencia a una dualidad vocacional, y en algunos casos incluso a una triple identidad, que se manifiesta entre sus roles como artistas, docentes e investigadores, y la influencia de estos en la forma en que gestionan la evaluación de aprendizajes en las asignaturas que imparten en la formación de futuros maestros. Los informantes comparten su identidad de docentes con la de artistas e investigadores, tal y como se desprende de los argumentos siguientes: "Yo me veo como un artista que comparte sus conocimientos con los demás, es decir, soy artista y soy docente" (Entrevista Profesor FEYTS-UVal10 mayo 2014); "el perfil es el del que vimos en clase que es el artista-docente-investigador. Entonces son las tres figuras, porque el artista cuando crea investiga, el artista cuando da clases investiga, hay una especie de relación entre esas tres figuras" (Entrevista Profesora FEYTS-UVal21 mayo 2014).

Esta dualidad vocacional puede resultar importante a la hora de entender las prácticas evaluadoras que desarrollan en sus aulas dado que estas varían sustancialmente en función de la dimensión vocacional que prime en cada caso.

En segundo lugar, nuestros informantes mencionan la existencia de tensiones a la hora de equilibrar en su docencia la impartición de contenidos técnicos y la didáctica de esos contenidos.

O sea, la formación artística la considero clave, y también la formación didáctica; porque aquí en Educación hay que tener claro que no educamos a artistas o sea lo que hacemos es... cómo los alumnos pueden trabajar la educación artística en el aula. Entonces, yo una competencia artística es la tengo que tener, pero también una competencia en ¿Cómo enseñar? (Entrevista Profesora FEYTS-UVal21 mayo 2014).

Esta tensión evidencia la influencia que en su forma de evaluar tiene la importancia que cada docente brinda a la enseñanza aprendizaje de contenidos, generalmente de corte conceptual, frente a la didáctica de los mismos, más centrada en procedimientos y actitudes.

En tercer lugar, nuestros informantes reconocen la existencia de diferencias claras en la formación que se imparte en Educación Plástica en Primaria y en Secundaria. Atribuyen este hecho a las diferencias en la formación didáctica inicial con la que cuenta el profesorado de primaria y secundaria en nuestro país.

Que es diferente, que eso, en Secundaria... se enseñan sobre todo contenidos, entonces los especialistas (ahora bueno porque está el Máster) pero antes (yo me acuerdo del $C A P)$ la formación didáctica que tenían era casi nula y cuando entraban en el aula se encontraban con el pastel de que no tenían ni idea de cómo enfrentarse a los problemas del día a día en el aula. (Entrevista Profesora FEYTS-UVal21 mayo 2014). 
Como se ha comprobado en la legislación actual, los profesionales no especializados en la Educación Artística pueden ser docentes de estas asignaturas en la Secundaria. Las carencias en la formación didáctico-pedagógica de estos profesionales pueden constituir una de las razones por las que en Secundaria se enseñen y evalúen más contenidos técnicos que en la Primaria.

\subsection{LAS COMPETENCIAS DOCENTES Y ARTÍSTICAS DE LOS INFORMANTES}

En esta segunda declaración encontramos una serie de competencias clave que los propios informantes han puesto en valor y consideran que han de ser trabajadas, y, por tanto, evaluadas.

La primera de ellas es la creatividad, entendida tanto como capacidad, proceso y producto. Para nuestros informantes, la creatividad permite elaborar respuestas originales, novedosas, alternativas. En todas las profesiones tiene cabida un ejercicio creativo de las mismas. Respecto al alumno, para desarrollar su creatividad, se incide en la eliminación de obstáculos formales:

[...] la creatividad es una pella de barro que no tiene forma, le damos la forma que nosotros queremos por nuestros intereses. El que es artista su creatividad la va a volcar en crear ese concepto que es un poquito ambiguo muchas veces o abstracto quiero decir, que se llama la belleza. (Entrevista Profesor FEYTS-UVal10 mayo 2014).

Como hemos comprobado por las distintas evidencias de los informantes, la creatividad será una competencia que habrá que desarrollar y, por tanto, evaluar; para su desarrollo han de eliminarse los obstáculos formales, sirviendo de motivación el propio proceso, que es el fin de la evaluación de los aprendizajes.

Otra competencia mencionada por nuestros informantes es la sensibilidad, entendida esta como la capacidad de saber responder al lenguaje propio de lo plástico y visual, y de tener una intención estética. Mencionan igualmente que aunque existe un componente innato relacionado con la sensibilidad, esta se puede educar, y que determinados ambientes educativos son más propicios que otros para hacerlo. También advierten sobre las trabas formales al desarrollo de la sensibilidad que suponen los enfoques rígidos de evaluación o la rigidez temporal que implica la adquisición de competencias.

La sensibilidad es algo que se puede desarrollar, bajo mi punto de vista y de los estudios que encontramos esa es una capacidad que se puede desarrollar, lo que pasa es que volvemos a lo mismo, tienes que saber cómo enseñarla, que la sensibilidad no es el uso de materiales, sino que es una especie como de relación, crear como una serie de actitudes, valores, gustos, apreciaciones... es algo más. (Entrevista Profesora FEYTS-UVal21 mayo 2014).

La competencia comunicativa es otra de las competencias destacadas por nuestros informantes en lo relativo a la gestión de grupos y su dinamización. También en su vertiente de trabajo en equipo (comunicación entre docentes), así como competencia discente a desarrollar por el alumno. La propia dinámica de las clases contaría con un diálogo procesual y continuo entre el profesor y el alumno, aunque esta forma de hacer 
conlleve más lentitud en la aplicación del programa de una asignatura. Además, esta competencia adquiere gran importancia en la forma de evaluar los aprendizajes, puesto que la comunicación y el feedback entre profesorado y alumnado es imprescindible para poder comprobar los cambios o aprendizajes que se van adquiriendo y desarrollando: "Yo creo que sobre todo hacen falta herramientas comunicativas y de gestión de los grupos, de organización, desde mi punto de vista” (Entrevista profesor de Secundarial18 mayo 2014).

Otra competencia a la que nuestros informantes dan importancia es la inteligencia emocional, entendida como la gestión de emociones en pos del equilibrio emocional, una adecuada expresión de las mismas emociones y la elaboración estrategias de resolución de conflictos. Abogan, por tanto, por la promoción de la participación continua del alumnado como agente fundamental en el aula. A la hora de enfrentarse a la evaluación de los aprendizajes, nuestros informantes plantean la necesidad de tener en cuenta la forma en que el alumnado gestiona sus emociones a lo largo del proceso de enseñanza aprendizaje, y si supera los obstáculos con resiliencia, etc.

Bueno, algunas ya las he comentado en la pregunta de antes: ser buen comunicador, tener competencias en cuanto a inteligencia emocional, para poder comunicar no solamente en los contenidos, sino también en las emociones que los chavales tienen, viven en cada momento y poder resolver conflictos, no solamente atajando y mandando al chaval a jefatura de estudio. (Entrevista Profesor de Secundarial18 mayo 2014).

Por último, otra competencia que nuestros informantes creen que es importante en el proceso educativo es la autonomía. Es la competencia que permite transformar las ideas en actos. La que permite al alumnado asumir gran parte de la iniciativa a la hora de auto organizarse, por ejemplo, en la resolución de conflictos, sin una ayuda excesiva de su círculo más cercano. Nuestros informantes han manifestado prestar atención a si el alumnado actúa por iniciativa propia o si necesita continuamente de la ayuda del profesorado o de los demás compañeros o familias.

[...] sino intentando resolver los conflictos para que los alumnos aprendan a resolverlos; o, mejor aún, intentando buscar la forma que los chavales sean capaces de resolverlos, sin que el profesor sea el que dirija todo el proceso. (Entrevista Profesor de Secundaria\18 mayo 2014).

\subsection{FORMAS Y ESTRATEGIAS DE EVALUACIÓN}

La tercera declaración temática hace referencia a las formas y estrategias de evaluación que nuestros informantes implementan en sus aulas.

En primer lugar, encontramos que la totalidad de nuestros informantes defienden la evaluación continua y formativa, como estrategia definitoria de su práctica evaluadora. Parece haber acuerdo en el hecho de que la evaluación de procesos, más allá de la unicidad de la evaluación sobre resultados finales, es fundamental en esta área de conocimiento. No obstante, vuelven a salir a la luz los obstáculos con los que se encuentran los formadores de futuros docentes para realizar el tipo de evaluación que consideran más adecuada (los tiempos, las ratios, el currículo): "me encuentro con sus producciones, que han sido tuteladas, porque yo voy siguiendo la realización del trabajo en el aula, me gusta que sea en el aula" 
(Entrevista Profesor FEyTS-Uva\10 mayo 2014); "procuro que lo hagan en la clase, pero dado los programas que tenemos, pues no puedes limitarte a eso, entonces si los traen hechos pues se evalúa igual el producto final" (Entrevista Profesor FEyTS-Uval10 mayo 2014).

Un segundo aspecto que nuestros informantes mencionan acerca de los procedimientos de la evaluación que implementan, es el uso del feedback y el diálogo. No obstante, encuentran reticencias por parte del alumnado que en muchas ocasiones solo busca la nota que califique su producto final. Por otro lado, el profesorado universitario y de secundaria admite no enseñar explícitamente cómo se evalúa a su alumnado. Este aspecto es realmente interesante para nuestro estudio, dado que nos permite observar una carencia curricular que hace que en numerosas ocasiones los futuros docentes imiten la manera en que ellos han sido evaluados. No obstante, uno de nuestros informantes, una profesora universitaria estadounidense, menciona que en su caso sí que enseña explícitamente cómo evaluar. "pequeñas evaluaciones y además de un tú a tú con el alumno y oral porque dice que es lo que más funciona” (Entrevista Profesora FEYTS-UVal21 mayo 2014).

\begin{abstract}
¿Desde el punto de vista filosófico? Habitualmente no pongo una nota a un trabajo doy feedback textual o verbal, pero feedback en lugar de una nota, que es algo muy complicado para ellos porque ellos quieren tener una nota. (Entrevista Profesora universitaria USAI2 mayo 2014).

[...] yo quiero que ellos reenfoquen un poco su labor como estudiantes y se centren en la calidad de lo que están aprendiendo y en apropiarse de su aprendizaje en lugar de que se centren en la nota final. (Entrevista Profesora universitaria USAl2 mayo 2014). [...] en el micro assessment lo que haces es olvidarte un poco de la nota final y centrarte en cómo van sucediendo las cosas en el aula, ellos lo van haciendo consigo mismos, aprenden a cómo evaluar y a evaluarse a símismos, sí, pero además desde una doble vertiente, desde la disposición y el progreso del alumno y la tuya propia, en ese momento concreto en el que estás enseñando algo. (Entrevista Profesora universitaria USAI2 mayo 2014).
\end{abstract}

En tercer lugar, destacamos las reticencias mostradas por nuestros informantes a la hora de implementar sistemas numéricos de evaluación (calificación) como herramienta principal de evaluación de los aprendizajes de su alumnado. Algunos de ellos plantean cuestiones morales y de justicia ligados a la trascendencia histórica de la nota numérica. Otros mencionan que el sistema les ha atrapado en su quehacer como docentes y se encuentran con un problema del que no pueden escapar y difícil de solucionar, ya que están obligados al final a calificar a cada alumno: "No son una evaluación de poner nota, ojo, cuando hablas de evaluación yo hablo de valoración, sí, no pongo una nota numérica" (Entrevista Profesora FEYTS-UVal21 mayo 2014).

[...] y otra evaluación que es la que debo hacer por mi profesión, porque los alumnos tienen que tener una calificación a lo que hacen. Para mí esa información no es útil, o muy poco, la tengo que poner, pero realmente nunca he estado cómodo con ese sistema”. (Entrevista Profesor de Secundaria\18 mayo 2014).

“[...] y siempre hay y en esto no nos podemos escapar en Arte nunca, los que evaluamos Arte un ingrediente que es subjetivo, difícil adjudicarle una nota numérica" (Elementos 
internos\Entrevista Manuel\10 mayo 2014); "yo no les quiero dar una nota, que es algo muy complicado para ellos, porque ellos quieren tener una nota" (Entrevista Profesora universitaria USA12 mayo 2014).

El cuarto y último aspecto de esta declaración temática está relacionado con la evaluación mediante ítems diferenciales que maticen específicamente los aprendizajes a mejorar. Cuanto se utilizan, por ejemplo, rúbricas para evaluar por competencias, estas han de subdividirse en diferentes dimensiones que permitan valorar si una competencia ha sido adquirida. Para valorar el nivel de adquisición de una competencia, se debe elaborar una serie de indicadores que la hagan medible. A pesar de su relevancia, es un aspecto que nuestros informantes no realizan, a pesar de usar rúbricas como estrategia de evaluación.

Por otro lado, en las entrevistas realizadas se han mencionado otras estrategias novedosas como el micro assessment (micro-evaluación) (Stake \& Munson, 2008), que pretende que cada proceso de evaluación sea una experiencia única e individualizada, aglutinando elementos formales (contenidos) y otros elementos de carácter informal (actitudes, emociones, competencias, destrezas, etc.) presentes en un proceso educativo en el campo de la educación artística. Esta aproximación requiere que el formador de futuros maestros dedique parte del currículum a enseñar a evaluar en el área. Esta forma de evaluación difiere además de aproximaciones más tradicionales, en que se aleja de procedimientos estandarizados de evaluación, buscando fórmulas que tienen que ver con la interacción situacional entre el formador y el futuro docente (por ejemplo, uso de experiencias de la vida cotidiana del discente).

Finalmente, en esta declaración temática, queremos destacar que algunos de nuestros informantes plantean el uso de una evaluación de aprendizajes heterodoxa, en periodos y marcos no lectivos, sin el apremio del sistema oficial, quizá bastante más motivador para el alumnado, aunque mucho más difícil de alinear con el sistema oficial de evaluación: "A la hora de evaluar, tengo una serie de ítems, que van de lo más formal, de lo más básico a lo más conceptual" (Entrevista Profesor FEyTS-Uval10 mayo 2014); "yo intento no utilizar calificaciones numéricas en los trabajos de clase; los valoro estableciendo una serie de ítems" (Entrevista Maestra de Primarial26 diciembre 2013).

[...] aprender a preguntar mejor a los niños para que entiendan mejor lo que les estás preguntando, entonces, dice que muchas de las cosas que se incluyen en las rúbricas habituales de assessment ella las utiliza porque le funcionan, pero que tienen mucho más que ver con esa concepción general de entender lo que está allí como una experiencia única. (Entrevista Profesora universitaria USAI2 mayo 2014).

\subsection{CURRÍCULO EN EDUCACIÓN ARTÍSTICA}

En nuestro estudio de caso los informantes entrevistados han valorado una serie de competencias docentes y artísticas para poder realizar una adecuada evaluación de aprendizajes. Sin embargo, en el análisis de la legislación actual aparecen competencias que no han sido tenidas en cuenta por los informantes y viceversa. Si unificamos las competencias que aparecen en la legislación con las que han sido propuestas por nuestros informantes, obtenemos la particular lista que mostramos a continuación: desarrollo de la creatividad; desarrollo de la sensibilidad; desarrollo de la competencia comunicativa; desarrollo de la inteligencia emocional; desarrollo de la autonomía personal; conocimientos 
y procedimientos relacionados con la práctica educativa, técnicas, fundamentos, etc.; desarrollo de habilidades relacionadas con la praxis (interpretar, saber buscar información, sentido y finalidad de la práctica, etc.) y; desarrollo de un compromiso ético.

Dentro de esta declaración temática, resulta también interesante mencionar las siguientes paradojas que hemos encontrado.

La primera podría formularse sucintamente como: "El currículo no nos gusta, sigamos el currículo". Es decir, nuestros informantes cumplen las orientaciones propuestas por el currículo, sin embargo, declaran abiertamente encontrarse con contradicciones en la práctica docente. Los docentes se esfuerzan por seguir las competencias del currículo, pero los centros educativos no poseen recursos pedagógicos para desarrollar correctamente dichas competencias, por lo cual el seguimiento del currículo es individualista y apoyado en competencias más generales como la creatividad.

Un segundo aspecto a este respecto es la potenciación de las competencias transversales, también en educación artística. Nuestro estudio pone de manifiesto la gran relevancia que los informantes dan a las competencias transversales en educación plástica, de entre las que destacan: aprender a aprender, competencia comunicativa, autonomía personal y autonomía tutelada de los grupos, etc.

La integración de diferentes valores simbólicos, culturales y sociales puede servirnos para aplicar los contenidos artísticos a otras áreas $\mathrm{y}$, por lo tanto, que esta disciplina adquiera el lugar que le corresponde con relación al resto de las materias curriculares "El arte es un campo de conocimiento que se conecta con otras áreas dentro del currículo y que requiere de docentes con nuevos perfiles" (Jiménez, Aguirre \& Pimentel, 2009, p. 11).

Otro aspecto relacionado con la estructura del currículum oficial con el que nuestros informantes se enfrentan, es el distanciamiento de los elaboradores de las normas respecto al conocimiento in situ y la dinámica práctica de la enseñanza real.

Respecto a las metodologías de evaluación, la mayoría de nuestros informantes reconocen evaluar los procesos y las actitudes más que los productos finales, aunque también reconocen que estos deben tenerse en cuenta para dar respuesta a los requisitos del sistema educativo actual. Nuevamente, los docentes intentan seguir las orientaciones curriculares sobre la evaluación de aprendizajes en E. Plástica (también de la E. Patrimonial), sin embargo, y aquí encontramos la paradoja, tampoco el currículo desarrolla la metodología específica para esa evaluación, con lo cual las adaptaciones de dichas evaluaciones son también individualistas. El currículo expresa qué hay que evaluar, pero no dice cómo evaluarlo.

La evaluación de procesos y actitudes se recoge solamente de forma genérica en la norma. Por el contrario, la crítica viene por el excesivo peso que siguen teniendo en la misma los exámenes tradicionales y la secuenciación estricta de los contenidos y las pruebas.

La segunda paradoja tiene que ver con la convicción general de que la evaluación ha de centrarse en los procesos y actitudes, pero no se sabe bien cómo. Es decir, hay una gran distancia entre las convicciones teórico-personales-profesionales de los docentes y el déficit de metodologías disponibles en la praxis, para evaluar competencias en educación artística. El currículum no explica la forma de realizar la evaluación de los procesos y actitudes. Los docentes en la práctica siguen una cierta secuenciación: primero hay un convencimiento teórico del docente que genera métodos y una búsqueda individual de recursos (semi-anárquica, o poco documentada); posteriormente busca un apoyo en las 
innovaciones que parten de los diseños curriculares; y, por último, añade un feedback de su praxis específica, etc.

Analizando esta dinámica, se podría concluir que la evaluación es un proceso que parece descoordinado, sin circulación de información entre los investigadores, los legisladores, la dirección de los centros y los docentes, y mucho menos con las familias, los agentes sociales o los creadores artísticos.

La tercera paradoja está relacionada con la praxis del profesor artístico (también profesor de contenidos patrimoniales) que podríamos decir se ha instalado en el hacer de manera poco reflexiva. Parece que la costumbre ha ido calando hasta que en ciertos casos se duda de la necesidad de establecer puentes con la teoría y la metodología pedagógica.

\section{CONCLUSIONES Y TRABAJO FUTURO}

Tal y como mencionábamos al inicio de este trabajo, una de las intenciones del mismo pasa por intentar aplicar los resultados obtenidos al campo de la Educación Patrimonial. Para ello debemos matizar qué entendemos la Educación Patrimonial tal y como se plantea en Fontal (2016), quien nos invita a hacer un recorrido por los textos curriculares de las tres últimas décadas, en el que ha podido llegar a los siguientes planteamientos: a) Existe una estrecha vinculación entre Educación Artística y patrimonio en la Educación Primaria; b) La formación de maestros debe incluir la formación en Educación Patrimonial; c) El área universitaria de Didáctica de la Expresión Plástica ha ido consolidando una línea de investigación en Educación Patrimonial; d) El Plan Nacional de Educación y Patrimonio (2013, PNEyP) permitirá consolidar la investigación, innovación y formación en Educación Patrimonial durante la próxima década; e) La Educación Patrimonial es rentable en términos sociales, culturales e identitarios.

Nuestro estudio de caso se ha centrado en el ámbito de la Educación Artística, que como menciona igualmente Fontal (2016) "tiene una estrecha vinculación con la Educación Patrimonial en Primaria. Tanto la Educación Artística como las Ciencias Sociales tienen una vinculación demostrada con la Educación Patrimonial, ya que son ambas quienes desarrollan contenidos sobre el patrimonio" (p. 116).

Como la Educación Plástica y Visual pertenecen al ámbito de la Educación Artística, y esta a la Educación Patrimonial, podríamos extrapolar nuestras conclusiones al terreno abonado de los debates teóricos y sociales del tratamiento del patrimonio en la Educación Primaria.

Así pues, las conclusiones generales emanadas del estudio de caso desarrollado, que podrían aplicar al campo de la Educación Patrimonial serían:

- Los informantes destacan el compromiso humano como eje de una motivación progresiva y continua en la profesión docente, cuestión que podría aplicar fácilmente al desarrollo curricular de la Educación Patrimonial en primaria.

- Por otro lado, los resultados de nuestro estudio muestran que resultaría recomendable que los maestros, profesores y formadores de futuros maestros en Educación Artística, equilibrasen los contenidos curriculares con la didáctica de esos contenidos, para fomentar una educación integral donde la evaluación de los aprendizajes pueda ser global y más equitativa. La Educación Patrimonial podría 
beneficiarse igualmente de este principio emanado de las entrevistas con nuestros informantes.

- Nuestros informantes han valorado como importantes un conjunto plural de competencias para el desarrollo de la educación artística. Unas son artísticas y otras de carácter didáctico-docente, tal y como mostramos en la sección anterior. No obstante, y aunque todas ellas merecen igual atención, los docentes ponen especial énfasis en el desarrollo y la promoción de competencias transversales, investigadoras, sistémicas y comunitarias, todas ellas ligadas a la pedagogía más contemporánea. Este hecho resulta interesante dado el impacto que tiene a la hora de establecer estrategias y recursos para evaluarlas. La propia Educación Patrimonial, considerada eminentemente como un área transversal, podría beneficiarse de este hecho al asumir que uno de sus componentes esenciales es la educación artística, tal y como se muestra en la Figura 3.

Figura 3. Educación patrimonial (Fontal, 2016)

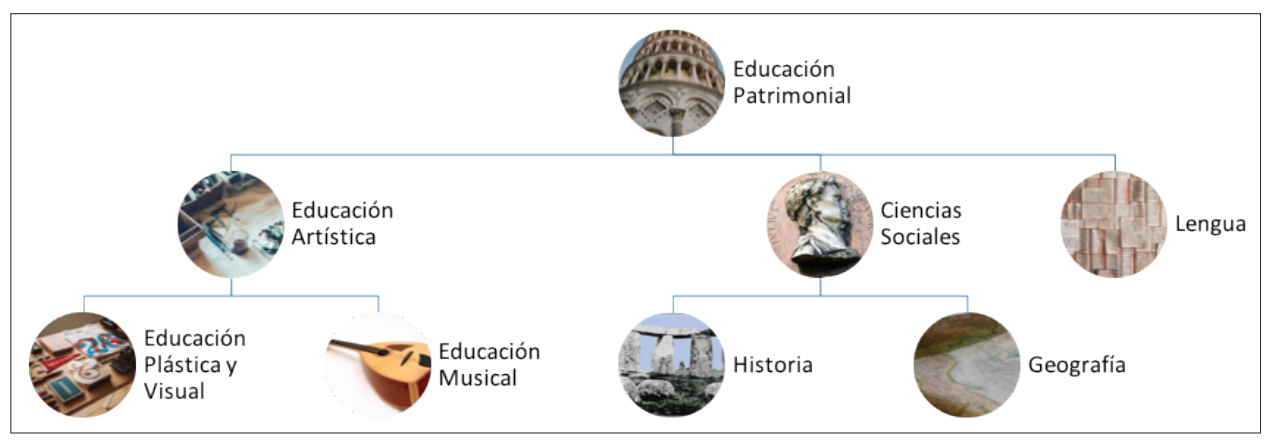

Fuente: adaptado de Fontal (2016).

La figura anterior muestra las tres áreas curriculares más importantes dentro de la Educación Patrimonial, aunque dado su carácter transversal, podría abarcar otras.

A este respecto, y dentro del trabajo futuro que podría emanar de estudios como el planteado en este artículo, sería interesante plantear estudios en profundidad sobre las concepciones de patrimonio que tiene el profesorado y alumnado de las materias que conforman la Educación Patrimonial. Este tipo de investigaciones permitiría vislumbrar la relación histórica y sociológica del diálogo entre el patrimonio y la escuela española a lo largo de las últimas décadas. Por ejemplo, se podrían promover estudios sobre el enfrentamiento entre un legado histórico concebido como corpus rígido proclive al adoctrinamiento del educando, y la visión contemporánea del patrimonio como exploración multifacética y global de las manifestaciones artísticas.

Igualmente, se podrían promover estudios centrados en el análisis de la forma en que la praxis docente refleja la inclusión de aspectos relacionados con el patrimonio en los currículos, la evaluación por competencias y/o la formación inicial que los futuros docentes tienen a este respecto. 
En nuestro trabajo hemos analizado la tensión existente entre las demandas curriculares establecidas por la legislación actual, las inercias de las instituciones educativas y las prácticas docentes innovadoras. Tal y como muestran algunos de los datos presentados en la sección de resultados, la rigidez propia del currículo oficial, así como la escasez de recursos existentes para su aplicación en lo relativo a la evaluación de los aprendizajes, hacen que los docentes aboguen por procesos de evaluación/calificación de corte sumativo que muchas veces implican una escisión entre las distintas asignaturas que conforman la Educación Artística. Desde nuestro punto de vista, la Educación Patrimonial podría ayudar a solventar estas tensiones desde tres aspectos fundamentales:

En primer lugar, ayudando a promover la interacción entre asignaturas y/o áreas de conocimiento. En segundo término, facilitando la permeabilidad de la Escuela al medio social, fomentando así la idea de que el legado histórico pertenece a todas las generaciones y que ha de ser aprehendido por el educando en cualquier espacio social donde se manifieste. Y tercera, la de ayudar a entender al alumno como participante además de observador o sujeto pasivo de las manifestaciones artísticas.

\section{REFERENCIAS BIBLIOGRÁFICAS}

Bamford, A. (2006). The wow factor: Global research compendium on the impact of the arts in education. Münster: Waxmann Verlag.

Bauman, Z. (2007). Tiempos líquidos: vivir en una época de incertidumbre. Barcelona: Tusquets.

BOE núm. 106 de 4/5/2006, páginas 17158 a 17207. LOE: Ley Orgánica 2/2006, de 3 de mayo, de Educación.

BOE núm. 117 de 20/06/2014, páginas 44181 a 44768. ORDEN EDU/519/2014, de 17 de junio, por la que se establece el currículo y se regula la implantación, evaluación y desarrollo de la educación primaria en la Comunidad de Castilla y León.

BOE núm. 295 de 10/12/2013, páginas 97858 a 97921. LOMCE: Ley Orgánica 8/2013, de 9 de diciembre, para la mejora de la calidad educativa.

Bruner, J. (1972). El Proceso de educación. México: Ed. Uteha.

Creswell, J. W. (2013). Research design: Qualitative, quantitative, and mixed methods approaches. EE. UU.: Sage publications.

Dewey, J. (1900). Psychology and social practice. Psychological review, 7(2), 105-124.

Domingo, M., Fontal, O., \& Ballesteros, P. (2013). Plan Nacional de Educación y Patrimonio. Madrid: Ministerio de Educación, Cultura y Deporte: Secretaría de Estado de Cultura.

Efland, A. D. (2002). Una historia de la educación del arte. Tendencias intelectuales y sociales en la enseñanza de las artes visuales. Barcelona: Paidós.

Eisner, E. W. (1995). Educar la visión artística. Barcelona: Paidós Ibérica.

Fontal, O. (2016). El patrimonio a través de la educación artística en la etapa de primaria. Arte, Individuo y Sociedad, 28(1), 105-120.

Formichella, M. M., \& London, S. (2005). Reflexiones acerca de la noción de empleabilidad. Argentina: Consejo Nacional de Investigaciones Científicas y Técnicas.

Greer, D. F. (1984). Industrial organization and public policy. New York: Macmillan Publishing Company.

Jiménez, L., Aguirre, I., \& Pimentel, L. G. (2009). Educación artística, cultura y ciudadanía. Organización de Estados Iberoamericanos para la Educación, la Ciencia y la Cultura. Madrid: Fundación Santillana.

Jorrín-Abellán, I. (2016). Hopscotch Building: A Model for the Generation of Qualitative Research 
Designs. Georgia Educational Researcher 13(1). doi:10.20429/ger.2016.130104

Kincheloe, J. L., \& Steinberg, S. R. (1997). Changing multiculturalism. England: Open University.

Lave, J., \& Wenger, E. (1991). Situated learning: Legitimate peripheral participation. Cambridge: Cambridge University Press.

Legrand, J. (1970). Guía del trabajo cotidiano en el aula. Buenos Aires: Kapelusz.

Marín-Viadel, R. (1987). ¿Medir los resultados o comprender los procesos? Icónica: revista de las artes visuales, didáctica e investigación, 9, 41-62.

Mishra, P., \& Koehler, M. J. (2006). Technological pedagogical content knowledge: A framework for teacher knowledge. Teachers College Record, 108(6), 1017-1054.

Perrenoud, P. (2009). Enfoque por competencias, ¿una respuesta al fracaso escolar? Revista Interuniversitaria de Pedagogía Social, 16, 45-64.

Piaget, L., Lorenz, K., \& Erikson, E. (1992). Juego y desarrollo. Méjico: Grijalbo.

Robinson, K. 1999. Culture, Creativity and the Young: Developing Public Policy (Cultural Policies Research and Development Unit Policy Note $\mathrm{N}^{\circ} 2$ ). Estrasburgo: Consejo de Europa.

Salgado, E. (2012). Enseñanza para la comprensión en la educación superior: la experiencia de una universidad costarricense. Revista iberoamericana de educación superior, 3(8), 34-50.

Sánchez-Macías, I. C. (2014). La evaluación por competencias en Educación Plástica en Primaria: Un estudio de caso (Trabajo Fin de Máster). Universidad de Valladolid, Facultad de Educación y Trabajo Social, Valladolid.

Sharp, C., \& Le Métais, J. (2000). The Arts, Creativity and Cultural Education: An International Perspective. London: Qualifications and Curriculum Authority.

Stake, R. E. (1995). The Art of Case Study Research. Thousand Oaks: Sage Publications.

Stake, R., \& Munson, A. (2008). Qualitative assessment of arts education. Arts \& Education Policy Review, 109(6), 13-22.

Tikunoff, W. J., \& Griffin, G. A. (1979). Interactive research and development on teaching study: Final report. San Francisco: Far West Laboratory for Educational Research and Development.

Vygotsky, L. S. (1988). Aprendizagem e desenvolvimento intelectual na idade escolar. In Vigotskii, L. S., Lúria, A. R., \& Leontiev, A. N., Linguagem, desenvolvimento e aprendizagem (pp. 59-83). São Paulo: Ícone: EDUSP.

Yazan, B. (2015). Three approaches to case study methods in education: Yin, Merriam, and Stake. The Qualitative Report, 20(2), 134-152. 